\title{
Transdisciplinary Research and Action to Stop Buruli Ulcer: A case Study from Philanthropy
}

\author{
Susanna Hausmann-Muela and Ann-Marie Sevcsik
}

\section{Introduction}

Over the past decades, advances in Buruli ulcer (BU) research and control have been substantially shaped by philanthropic and civil society engagement. It has been a long journey for advocates to attract public attention and funding for BU. In 1998, the World Health Organization (WHO) established the Global Buruli Ulcer Initiative, supported by the Nippon Foundation. A first international conference on BU, organized by WHO, led to the 1999 Yamoussoukro Declaration, which brought greater public awareness to the disease, for the first time [1].

Intensified research and control activities started thereafter. In 2004, the World Health Assembly adopted a resolution on BU, which called upon the international community, organizations and bodies of the United Nations system, donors, nongovernmental organizations, foundations and research institutions to cooperate directly with disease-endemic countries in order to strengthen control and research activities; and to develop partnerships and to foster collaboration with organizations and programs involved in health-system development, in order to ensure that effective interventions can reach all those in need [2].

Despite increased attention and the coordinated efforts by the WHO-Global Buruli Ulcer Initiative, research and control activities remained highly underfunded, with advances limited. In 2007, the UBS Optimus Foundation (UBS-OF) identified BU as a disease where a relatively small investment had the potential to make a big difference. With the goal to catalyse a transdisciplinary consortium bundling BU

Susanna Hausmann-Muela was responsible for the UBS Optimus Foundation led Stop Buruli Initiative from 2007-2011, Ann-Marie Sevcsik from 2012-2018.

S. Hausmann-Muela $(\triangle)$

Fondation Botnar, Basel, Switzerland

e-mail: shausmann@fondationbotnar.org

A.-M. Sevcsik

UBS Optimus Foundation, Zürich, Switzerland

(C) The Author(s) 2019

G. Pluschke, K. Röltgen (eds.), Buruli Ulcer,

https://doi.org/10.1007/978-3-030-11114-4_16 
research into a concerted effort, the UBS-OF engaged researchers from different disciplines and countries, with a prominent role of endemic countries and good links to national BU control programs.

Philanthropic engagement aspires for innovation and impact. This chapter takes a critical look back at the whys, hows, and major results of the Stop Buruli Initiative initiated by the UBS-OF and partially co-funded by the Medicor Foundation. It discusses the long-term and comprehensive approach of the UBS-OF in research and control of $\mathrm{BU}$, and the lessons learned for achieving the aspiration of innovation and impact.

\section{The Beginnings: Six Reasons Why}

\subsection{Reason \# 1: Lack of Funding Incentives}

BU is one of the Neglected Tropical Diseases (NTDs) listed under the WHO classification system. These disease conditions disproportionately affect populations living in tropical and sub-tropical areas and people living in poverty.

Research and development efforts for BU were marginal. Like for many other NTDs, industry has no incentive to invest in products such as diagnostics, medicines, vaccines and other control tools defined by global health needs or public priorities and targeting populations and governments with low purchasing power. According to G-Finder data, total investment in BU research by the private sector between 2007 and 2016 was merely around USD 300,000.

Furthermore, philanthropic and public research funding for BU has been far lower than for other tropical diseases. Between 2007 and 2016, BU research funding amounted to as little as 38 million USD in total. Slightly more than one third (14 million USD) originated from philanthropic sources, with the remaining (24 million USD) from the public sector, which majorly invested in basic or vaccine research. The biggest single commitment was the 2010-2013, EU-supported project to develop a vaccine protecting against BU (BuruliVac).

The lack of interest and funding for BU also affects the academic world. BU remains one of the least studied diseases because this is not where researchers can make a great academic career. Little attention means small academic impact, difficulties to publish in high-ranking journals and to acquire research funding. For a foundation with relatively small grant-making resources, this 'niche' situation was a compelling argument to invest in BU.

\subsection{Reason \#2: A Disease of Social Justice}

The second compelling argument was based on social justice and a desire for global health equity. The neglect of the disease goes along with the neglect of the affected populations. BU affects the most vulnerable and voiceless people. Children living in remote and poverty-stricken areas of Africa are suffering the most. 
Findings from epidemiological studies indicated that the BU burden in Africa was underestimated [3] and solid world-wide prevalence data were not available. BU was reported in 33 countries, but not all endemic countries regularly reported case numbers to the WHO and in some countries, endemic regions still have to be mapped. Assessment of the global disease burden was impeded by the remoteness and poverty of the most affected populations and countries. The Global Burden of disease study 2016, the most comprehensive worldwide observational epidemiological study, did not include estimates on BU burden.

Organizations working on BU were confronted with a spiral of neglect: lack of epidemiological data veiled the evidence of the real dimensions of the disease. This, in turn, compromised attention and support, which hindered the collection of more robust and comprehensive evidence. This, characteristic for most neglected diseases, is a matter of social justice, exacerbating the shadowy existence of the victims of the disease.

\subsection{Reason \#3: A Disease with Devastating Consequences}

The poorest populations, lacking access to health care, were the least served. Even though case fatality may be low, the severity and chronic nature of the disease often leads to devastating consequences of BU for the individual, their families and the affected communities. When diagnosed and treated early, BU can be a wellmanageable disease. However, at the time, the majority of patients did not seek biomedical treatment until the condition was in later stages. Massive extension of BU lesions and delayed wound healing often causes irreversible physical disability due to deformities on arms, legs, trunks and in the face. Alone the painful changing of wound dressings already makes the disease a human tragedy for all affected. Stigma, discrimination, and the need for long-term hospital stays far away from home aggravated the social and economic conditions for affected households. The cost burden due to long-term treatments far from home and productivity loss of BU patients, and of parents accompanying children during hospital stays, can amount to catastrophic levels for the household economy, not seldom leading to abandonment of patients by the family [4].

A human-rights based perspective justified a global response to BU, but attention failed to increase.

\subsection{Reason \#4: The Pioneer Effect}

While the needs-driven perspective to reduce inequity, social injustice and human suffering was convincing for philanthropic support, there was also the argument of likelihood for success. Philanthropic support tends to be open to taking risks, but only if these are calculated. Investment into BU research was likely to show a positive benefit/risk balance. 
$\mathrm{BU}$, coined the mysterious disease, was characterized by a great vacuum of knowledge and little understanding by researchers, implementers, and affected persons themselves. It was a disease where nearly nothing was known regarding transmission and epidemiology, where clinical presentation was difficult to interpret even by experts, where diagnostic procedures were complicated, costly and often leading to false results, where wide surgical excision of the affected tissue was the only recommended treatment option, where efficacy of antibiotic therapies was debated, where patients often had no access to treatment and where it was not clear how to reduce the risk of acquiring the disease.

To start to bring light into such nearly total darkness is a way to become a pioneer in an area where very few others are engaged but may join the bandwagon after they see success. The opportunity to be at the forefront of research and disease control, and to act as a catalyst by convening a variety of stakeholders and partners, was one of the most convincing arguments for the UBS-OF to engage in a multi-disciplinary BU initiative.

This pioneering effect in combination with the prospect that relatively small investments can make a real difference for many patients and their families was a powerful argument. The reason why little money could lead to big impact was also related to the fact that there was no need for big investments into new infrastructure, since much of the activities could piggyback on existing and well-established research and health care structures.

\subsection{Reason \#5: Existing Critical Mass of Researchers and Implementing Agencies}

Research groups working on BU already existed, although the primary foci of researchers in the field were usually other mycobacterial diseases, like tuberculosis and leprosy, and not BU. Researchers with suitable expertise were therefore available and at the time when the Stop Buruli Initiative was launched, it was not the absence of scientific know-how or interest, but the lack of funding and coordination that impeded scientific progress.

A network analysis of institutions active in BU research at the time showed how small yet fragmented the scientific community was. Organizations active in BU research spanned all over the world, over four continents, with very diverse disciplines, expertise and experience.

The existence and diversity of this critical mass of researchers and institutions willing to engage in BU research was essential for rapid progress and potential for impact.

\subsection{Reason \#6: Scalability and Uptake}

Despite fragmentation, a network of collaborations between institutions from different affected countries and national disease control programs already existed. The 
network analysis of institutions and publications concluded that most BU research was quite recent, mainly starting in the 1990s, but fast-growing especially in the early years of 2000 when the WHO Global Buruli Disease Initiative was established. By the year 2007, nearly a dozen experts had published more than 50 articles, and roughly 20 institutions worldwide were involved in research, with a high density of inter-institutional collaborations. Research activities were on-going primarily in four countries with BU case reports: Australia, Benin, Cameroon and Ghana.

Existing longstanding institutional partnerships between these four countries and researchers in the USA, Belgium, and Switzerland were a key element for setting up the new consortium. The organizational anchoring and partnership-based collaborations with national Ministries of Health and National BU control programmes were crucial. This permitted scientists to connect with practitioners and to consolidate and translate research efforts into action. From the beginning, favourable conditions existed for access to patients and specimens, as well as a high likelihood of integrating new findings into policy guidelines and practice. The WHO Global Buruli Ulcer Initiative, with its annual meetings that brought together all relevant stakeholders from across the globe and the Technical Advisory Group that included many of the researchers, enabled translation of key findings into normative guidelines at the global level.

\subsection{Stop Buruli Initiative}

In sum, the decision to invest in BU research and control followed a set of principles and reflections. An initiative was defined by the UBS-OF as "a relevant and neglected field, with a potential for change, where the foundation can take a leading role." BU matched the four elements contained in the definition. It is a relevant disease, as emphasized by international declarations and a World Health Assembly adopted resolution in 2004; it is neglected, i.e. not in the spotlight and receiving scarce resources due to both market and policy failures; there is a potential for change by helping to develop standard know-how and tools for diagnostics and therapies that are being implemented throughout African and other affected areas; and the UBS-OF can take a leading role through convening key stakeholders, creating cohesion among them, and attracting new funders to get aligned in a common effort to address the problem.

\section{The Model: Transdisciplinary Research and Implementation}

In 2007, the UBS-OF invited a core group of institutions to develop a consortium proposal with an overall research agenda, under the guiding principle to lead from innovation over validation of the findings and interventions to public health application. The invitation specified the major lines by mentioning transmission, 
ecological, social and economic, as well as clinical aspects, and by considering a focus on developing applicable and affordable tools; an integrated multidisciplinary approach; research capacity strengthening, including technology transfer and training of researchers from BU endemic countries; strengthening of North-South and South-South partnerships.

The consortium partners initially included seven institutions, with key expertise in different fields, including the Swiss Tropical and Public Health Institute (SwissTPH), Switzerland (research coordinator); Fairmed (former Aide Aux Lépreux Emmaüs-Suisse (ALES)), Cameroon; Institute of Tropical Medicine (ITM), Belgium; Noguchi Memorial Institute for Medical Research (NMIMR), Ghana; Programme national de lutte contre l'ulcère de Buruli et la lèpre, Benin; University of Melbourne, Australia; and University of Tennessee, USA.

This was the start of a model for a transdisciplinary approach, linking research to implementation, building local capacity across disciplines and through co-creating knowledge leading to action and, ultimately, impact for patients, their families, and countries. The role of the UBS-OF was to bring the diverse stakeholders together and to point the way to generate the tools and solutions that were needed, but also to advocate for and to ensure the taking up of those tools under real life conditions.

\section{$4 \quad$ Key Elements for Advancing Transdisciplinarity}

\subsection{Transdisciplinarity Does Not 'Just Happen'}

Transdisciplinary research agendas are often specified as a goal in the context of research for development promoted by development agencies or philanthropic grants. But experience shows how difficult it is to work across disciplines, and to consider policy and practice as an integral part of the research agenda. This requires patience, persistence and willingness to adapt the course based on factors internal and external to the initiative.

One key element is to convene the right disciplines and partners, and to jointly discuss and develop the aims and actions. But multi-disciplinary consortia are, by definition, complex constructs embracing not only different disciplines, but also different institutional cultures. Such a research consortium is of paramount complexity, since the element of North-South partnerships with all its socio-politically rooted facets adds to the already complex nature of a multi-stakeholder collaboration. A research consortium does not develop in a neutral space. Wanted or not, explicit or implicit, power relations among different partners may lead to unequal decision-making and imbalanced resource allocations.

To bring all disciplines and different partners into alignment and to foster an effective and efficient collaboration requires an active orchestration of the partnership. Beyond the financial resources, it is the convening power and the agile management of an engaged, unbiased donor that brings added value to the functioning of a complex consortium. 
The development of the social sciences within the Stop Buruli consortium provides an illustrative example for the relevance of such an active and agile management approach. The consortium embraced research and action along four axes: disease transmission, diagnosis, treatment, and socio-economic and cultural aspects. The UBS-OF convened representatives from all different disciplines right from the beginning, and all partners showed great willingness to develop a joint effort along the four axes. But the social science axis started to lag behind, unable to integrate their research questions into the overall consortium agenda. The first social science proposal suggested a classic information, education, and communication approach with the aim to educate the population about signs and symptoms of BU, but ignored to consider the research priorities of the other axes. The misalignment of the social science research risked developing into a parallel direction, leading to a fragmentation of workstreams instead of the concerted action that had been envisioned.

\subsection{Governance Model}

The key to redirecting the social sciences towards a path of convergence with the other disciplines was not to request the researchers to rephrase and resubmit proposals, but to adapt the consortium's governance structure. The consortium had constituted a steering committee with representatives from the seven different partner organizations, but lacked a representative from a strong social science partner. In other words, the social scientists were within the research teams but lacked a voice at the higher level of decision-making, i.e. at the level of the steering committee.

The solution to the problem was to seek and include an eighth partner, and to add a senior-level social science representative to the steering committee. This is how, upon request of the UBS-OF, the Anthropology Unit of the University of Arizona joined the consortium. That way, the research directions discussed and decided by the steering committee gained in quality concerning transdisciplinarity.

The governance model of the Stop Buruli Consortium then consisted of eight members of the steering committee and for reasons of agility, a day to day management team of three rotating members, overseeing the different work packages along the four axes. The close relation of the steering committee with the national $\mathrm{BU}$ control programs in the three BU endemic African countries involved (Ghana, Benin, Cameroon) and with the WHO Technical Advisory Group for BU permitted flexibility for the consortium activities, but at the same time close interaction with the Ministries of Health for collecting data and developing recommendations and guidelines, nationally and globally. The regular WHO Meetings on BU control and research served as an important platform for exchange with other stakeholders, which enabled results from the research activities to be brought into the broader context and influence policy discussions. 


\section{$5 \quad$ Results}

The three funding cycles (2007-2018) powered by the UBS-OF brought important advancements to research and control of BU as well as educating a future generation of African scientists. The broad transdisciplinary focus of the consortium with the integrated action not only secured an efficient and effective use of the funds, but it also leveraged funding from other donors, which continue with relevant, concerted research and action to this day. As the planning for the third cycle began, it was recognized by the UBS-OF and the consortium members that important channels had been built across disciplines and new partnerships formed, including a product development partnership with the Foundation for Innovative New Diagnostics (FIND), but that a formal structure to facilitate that cross-talk was no longer needed.

The research output of the consortium led to more than 60 publications, training of 25 African graduate students, more than 9000 BU sufferers having received improved treatment and education, as well as three hands-on, field workshops in Africa that allowed for professional and practical training of young scientists by senior experts in laboratory-based and field research. The consolidated approach achieved considerable success along the four axes. The following sections outline some of the major achievements and future perspectives:

\subsection{Diagnosis}

Diagnosis is a major challenge in BU control. District hospitals often rely on clinical diagnosis only, which is very often inaccurate and complicated by other disease conditions with similar presentation as BU. There is over/under treatment as a result of misdiagnosis and poor surveillance of the disease due to absence of effective diagnostic services. It is therefore important that clinical diagnosis is supported by laboratory confirmation.

The diagnostic field recorded impressive progress. The consortium optimized the method of fine needle aspiration for improved collection of specimens from closed lesions [5]. An illustrative and open access video-guide of how-to-do-it and WHO supported teaching and training activities improved the accuracy of diagnosis undertaken by qualified health staff in BU affected countries. In addition, optimization of PCR-based diagnosis by ITM improved laboratory confirmation of clinical cases $[6,7]$.

However, PCR technology based diagnosis is expensive, requires time and experienced health staff and technology, which are only available at specialized BU reference laboratories, which are far away from the affected patients and communities. Therefore, high priority was given to the development of rapid, low-tech, sensitive and specific diagnostic tests for use at district hospital and at community levels.

The identification of a $M$. ulcerans protein suitable for the development of an antigen capture assay [8] allowed to develop a simple ELISA executable at district hospital level. In collaboration with the FIND and Abbott/Standard Diagnostics 
(SD), the monoclonal antibodies generated by SwissTPH are now used in further research aimed at the development of a point-of-care test. First prototypes of rapid diagnostic tests (RDTs) are under laboratory validation; if successful, these will pave the way for surveillance and early diagnosis of BU at the community and primary health care levels.

Another promising avenue for diagnostic test development is a technology known as loop-mediated isothermal amplification (LAMP) of pathogen DNA. This approach has recently been exploited in development of simple molecular tests for many infectious diseases, among them tuberculosis, sleeping sickness, leishmaniasis, malaria and Chagas disease. The Noguchi Memorial Institute for Medical Research (NMIMR) in Ghana and the Department for Infectious Diseases and Tropical Medicine (DITM) in Germany, in collaboration with FIND and other partners, are advancing the development of a Buruli LAMP test, which is highly accurate, and does require less specialised training or equipment than PCR.

The macrolide exotoxin mycolactone of $M$. ulcerans plays a crucial role in the pathogenesis of BU. Therefore major efforts have been made to raise mycolactonespecific antibodies. However, in spite of numerous attempts by several expert laboratories, generation of antibodies against mycolactone had failed for a long time. Based on quantitative structure-activity relationship analysis with synthetic mycolactones [9] SwissTPH has successfully developed a new immunization strategy, using a protein carrier conjugate of a synthetic truncated non-toxic mycolactone derivative. It has turned out that this formulation elicits mycolactone specific antibodies that neutralize the toxin [10]. This provides the basis for a new approach for BU vaccine design supported by the Medicor Foundation (see below). Furthermore, the M. ulcerans specific mycolactone may represent an ideal target for the development of a specific diagnostic test and the successful generation of mycolactonespecific antibodies has opened new options for diagnostic test development, an approach followed by SwissTPH in collaboration with FIND.

\section{$5.2 \quad$ Treatment}

The Stop Buruli consortium has also achieved highly relevant findings for BU treatment. Concerns that secondary lesions after streptomycin/rifampicin combination therapy given for 8 weeks were linked to relapses or failures of treatments could be discarded, supporting the $\mathrm{WHO}$ recommendations in the treatment guidelines for health workers.

A specific focus was given to wound care and healing. One of the findings showed that delay in wound healing may be related either to clinical deterioration, known as paradoxical reactions, or to secondary bacterial infections. It could also be shown by the NMIMR in collaboration with SwissTPH and the University of Melbourne that BU wounds are frequently super-infected with hospital-acquired multi-resistant bacteria [11-13]. 
The early pathogenesis of $\mathrm{BU}$ and the molecular mechanism of tissue necrosis caused by the toxin mycolactone produced by $M$. ulcerans were investigated [1416] providing important insights relevant for diagnosis and treatment.

Wound management practices at primary and secondary health care facilities in Ghana were investigated and recommendations for improvements made [17]. In Benin a decentralized community-based treatment program for BU was implemented and assessed by the national control program supported by the University of Arizona [18]. The impact evaluation found that community confidence in decentralized BU care was greatly enhanced by clinic staff who came to be seen as having expertise in the care of most chronic wounds. The pilot program further demonstrates the added value of integrated wound management for skin NTD control. The model developed in this pilot study may serve as a foundation and proof of concept for a larger community-based decentralized wound care agenda.

A major conclusion of the consortium was that BU control should be integrated into horizontal programs for the integrated diagnosis and treatment of tropical skin diseases. WHO advocates for this approach and the UBS-OF continues to support a pilot project in Benin and Ivory Coast that includes several Stop Buruli members as well as the NGO Anesvad.

\subsection{Transmission}

Despite intensive environmental studies carried out by the consortium, the exact mode of transmission of M. ulcerans still remains unknown. However, studies brought new insights which contribute to the better understanding of the puzzle of transmission. For example, certain correlations between environmental factors and BU incidence could be shown by researchers from the NMIMR, which confirmed behavioural risk factors for contracting the disease, such as farming in swampy areas or application of leaves on wounds [19]. Sero-epidemiological studies showed that exposure to $M$. ulcerans increases at an age of about 4 years. However, many exposed individuals do not develop clinical disease [20]. Findings in M. ulcerans infected possums in Australia led researchers from the Melbourne University to propose that humans might harbour M. ulcerans in their gastrointestinal tract and shed the bacterium in their faeces. A study with faecal samples of BU patients from Ghana concluded however, that the human gastrointestinal tract is unlikely to be a significant reservoir of $M$. ulcerans.

M. ulcerans DNA has been detected in many environmental, in particular aquatic sources, but how bacteria enter the skin tissue is not clear. The hypothesis of bacteria entering through open wounds was tested using a guinea pig model by researchers of the University of Tennessee. While injection of M. ulcerans into the skin led to the development of lesions, the researchers were unable to produce infection via topical application of $M$. ulcerans on open abrasions. These results suggest that BU is not likely to be due to passive entry of bacteria into deeper skin tissue via superficial skin abrasions [21]. 
Evidence also showed that M. ulcerans is growing in amoeba, but this does not seem to represent an important environmental reservoir. In Australia, possums were found to develop clinical BU, and to serve as an animal reservoir in the Buruli endemic region of Victoria, but no comparable reservoir was found in Africa. While M. ulcerans DNA was found in the faeces of BU-affected possums in Australia, no such DNA could be demonstrated in faecal samples of human patients in Ghana [22].

A breakthrough was achieved through whole genome sequencing analyses which provided insights into the diversity, population structure and evolutionary history of M. ulcerans. The identification of local clonal complexes revealed that transmission over large distances is rare [23-26].

All in all, transmission of BU remains an enigma despite many insights into details relevant for transmission.

\subsection{Socio-Cultural Aspects}

The social science axis of the consortium investigated the cultural perceptions of BU and socio-economic factors influencing BU health-seeking and decision making. Using a formative research approach, interventions to improve early access of BU patients to diagnosis and treatment were designed and validated. In Cameroon, a BU community of practice involving multiple community stakeholders from clinic staff and former patients to community health workers and traditional healers resulted in improvement of case identification, reduction in health care seeking delay and an increase in treatment adherence [23, 27]. The process of social science intervention and major lessons learned from the Bankim case study in Cameroon are described in detail in chapter "Social Science Contributions to BU Focused Health Service Research in West-Africa" of this book. In Benin, establishing a therapeutic community in an in-patient ward resulted in positive impact on health staff and patient relations [28]. In Ghana, enhanced capacity of health staff in antibiotic treatment and wound care and community-based surveillance, patients screening and referrals to medical treatment improved early detection of disease [29]. In all three countries, pilot studies showed how patient support, such as transport or food offers improved treatment-seeking and adherence. The active engagement of different stakeholders from the communities, including former patients and traditional healers, addressing real life problems of the affected and their families contributed to improved early treatment and management of BU cases. The social science contributions and outcomes of the community-based studies and interventions are described in detail in the aforementioned "Social Science" chapter of this book. 


\section{$6 \quad$ Lessons Learnt and Conclusions}

Transdisciplinary research and action approaches are promising and currently much promoted by many institutions. The scope is multidimensional, and the aim is to create impact based on research and evidence that is directly applicable to real-life conditions. The first important lesson to learn from the Stop Buruli Initiative is the key role of the funder as a convening partner of a diverse group of partners, bringing the right expertise to the table and facilitating co-development of research and intervention.

The second lesson, related to the complexity of multidimensionality, is the relevance of time. Collaborating across disciplines is challenging, and requires sufficient space and time for exchange and mutual learning. From the beginning, the Stop Buruli Initiative had a time horizon for support of 9-10 years, with 3-year cycles, and regular meetings. The long-term commitment permitted the consortium to adapt its priorities and approaches based on interim findings as well as to strengthen research capacity and to pave the way for sustainable solutions.

The organization of the overall activities into different work packages certainly helped to structure the research along clear topics, with defined milestones and deliverables for all work streams. However, the third important lesson that can be drawn from the consortium is the importance of good governance and an agile management of the donor. To develop, test and validate research results in the field, considering the political, social and economic conditions, is not a direct inputoutput-outcome equation, but requires dialogue and continuous adjustments. Such dialogue requires an institutionally neutral agency, which has the legitimacy, is able and sufficiently flexible to enable shared learning and to change course of action if needed. The experience of the social sciences' initial lack of integration illustrates how important the governance model with equal representation of different disciplines and partners is, and how flexible, responsive and patient a donor needs to be.

The fourth lesson to learn from the model is the weight of communication and advocacy for leverage. By actively reaching out to other potential donors, offering well-established and reliable platforms for collaboration, lines of research and action could be further expanded. Support by additional donors allowed to peruse promising outcomes and partnerships from the consortium leading to further transdisciplinary research projects.

The Medicor Foundation, a philanthropic foundation based in Triesen, Liechtenstein, supported over a period of 10 years multidisciplinary research on BU. In a first phase, biomedical and field research activities in Cameroon and Benin, aiming at the improvement of diagnosis and treatment of BU were facilitated. Motivated by promising results, Medicor supported in a second phase diagnostics and vaccine research focussed on the key virulence factor of M. ulcerans, the macrolide toxin mycolactone. Within a 6-year cooperation, early product development activities have generated concept validation and candidate products. These lead candidates are now handed over to other funders, such as FIND (see below), for full product development. In a third phase, the Medicor Foundation is currently 
supporting a project aiming at the demonstration that thermotherapy of BU can be successfully implemented at health post level.

To consider funding, it was important for the Medicor Foundation, (1) to work with committed, professional, trusted and experienced partners and (2) that specific research projects were defined that were expected to generate with limited funding results within a period of 2-3 years. An important conclusion of the 10-year experience with research support for BU is that on the other hand only a long-term commitment covering several project phases can generate tangible results.

The Foundation for Innovative New Diagnostics (FIND) has supported development of diagnostics for BU since 2014, with funding from various sources, including UBS-OF, the Swiss Agency for Development Cooperation (SDC), the German Federal Ministry of Education and Research through KfW, and recently Anesvad.

The network of partnerships established through the Stop Buruli Consortium contributed immensely in identification of the unmet diagnostic needs for BU, development of the FIND strategy for the disease, and access to clinical samples for use in test validation.

In conclusion, the coordinated effort advanced through the model of the Stop Buruli consortium led to a substantial push in BU research and control. While the big philanthropic organizations are more prone to address big societal problems, smaller foundations are well positioned to address 'niche' problems that remain otherwise unaddressed, but where they can have a big impact, if well-managed and with the right partners in place, both in their niche and also serving as a model.

\section{References}

1. WHO (2018) History of the GBUI. http://www.who.int/buruli/gbui/en/. Accessed 08 Jun 2018

2. Resolution WHA57.1 (2004) Surveillance and control of Mycobacterium ulcerans disease (Buruli ulcer). In: Fifty-seventh world health assembly, Geneva, 17-22 May 2004. Resolutions and decisions (WHA57/2004/REC/1). World Health Organization, Geneva, pp 1-2

3. Röltgen K, Pluschke G (2015) Epidemiology and disease burden of Buruli ulcer: a review. Res Rep Trop Med 6:59-73. https://doi.org/10.2147/RRTM.S62026

4. Peeters Grietens K, Um Boock A, Peeters H, Hausmann-Muela S, Toomer E, Muela Ribera J (2008) "It is me who endures but my family that suffers": social isolation as a consequence of the household cost burden of Buruli ulcer free of charge hospital treatment. PLoS Negl Trop Dis 2(10):e321. https://doi.org/10.1371/journal.pntd.0000321

5. Eddyani M, Fraga AG, Schmitt F, Uwizeye C, Fissette K, Johnson C, Aguiar J, Sopoh G, Barogui Y, Meyers WM, Pedrosa J, Portaels F (2009) Fine-needle aspiration, an efficient sampling technique for bacteriological diagnosis of nonulcerative Buruli ulcer. J Clin Microbiol 47:1700-1704. https://doi.org/10.1128/JCM.00197-09

6. Affolabi D, Sanoussi N, Vandelannoote K, Odoun M, Faïhun F, Sopoh G, Anagonou S, Portaels F, Eddyani M (2012) Effects of decontamination, DNA extraction, and amplification procedures on the molecular diagnosis of Mycobacterium ulcerans disease (Buruli ulcer). J Clin Microbiol 50:1195-1198. https://doi.org/10.1128/JCM.05592-11

7. Eddyani M, Lavender C, de Rijk WB, Bomans P, Fyfe J, de Jong B, Portaels F (2014) Multicenter external quality assessment program for PCR detection of Mycobacterium ulcerans in clinical and environmental specimens. PLoS One 9:e89407. https://doi.org/10.1371/ journal.pone.0089407 
8. Dreyer A, Röltgen K, Dangy JP, Ruf MT, Scherr N, Bolz M, Tobias NJ, Moes C, Vettiger A, Stinear TP, Pluschke G (2015) Identification of the Mycobacterium ulcerans protein MUL_3720 as a promising target for the development of a diagnostic test for Buruli ulcer. PLoS Negl Trop Dis 9:e0003477. https://doi.org/10.1371/journal.pntd.0003477

9. Scherr N, Gersbach P, Dangy J-P, Bomio C, Li J, Altmann K-H, Pluschke G (2013) Structureactivity relationship studies on the macrolide exotoxin mycolactone of Mycobacterium ulcerans. PLoS Negl Trop Dis 7:e2143. https://doi.org/10.1371/journal.pntd.0002143

10. Dangy J-P, Scherr N, Gersbach P, Hug MN, Bieri R, Bomio C, Li J, Huber S, Altmann K-H, Pluschke G (2016) Antibody-mediated neutralization of the exotoxin mycolactone, the main virulence factor produced by Mycobacterium ulcerans. PLoS Negl Trop Dis 10:e0004808. https://doi.org/10.1371/journal.pntd.0004808

11. Kpeli G, Buultjens AH, Giulieri S, Owusu-Mireku E, Aboagye SY, Baines SL, Seemann T, Bulach D, Gonçalves da Silva A, Monk IR, Howden BP, Pluschke G, Yeboah-Manu D, Stinear T (2017) Genomic analysis of ST88 community-acquired methicillin resistant Staphylococcus aureus in Ghana. PeerJ 5:e3047. https://doi.org/10.7717/peerj.3047

12. Kpeli G, Darko Otchere I, Lamelas A, Buultjens AL, Bulach D, Baines SL, Seemann T, Giulieri S, Nakobu Z, Aboagye SY, Owusu-Mireku E, Pluschke G, Stinear TP, Yeboah-Manu D (2016) Possible healthcare-associated transmission as a cause of secondary infection and population structure of Staphylococcus aureus isolates from two wound treatment centres in Ghana. New Microbes New Infect 13:92-101. https://doi.org/10.1016/j.nmni.2016.07.001

13. Yeboah-Manu D, Kpeli GS, Ruf M-T, Asan-Ampah K, Quenin-Fosu K, Owusu-Mireku E, Paintsil A, Lamptey I, Anku B, Kwakye-Maclean C, Newman M, Pluschke G (2013) Secondary bacterial infections of Buruli ulcer lesions before and after chemotherapy with streptomycin and rifampicin. PLoS Negl Trop Dis 7:e2191. https://doi.org/10.1371/journal.pntd.0002191

14. Bieri R, Scherr N, Ruf M-T, Dangy J-P, Gersbach P, Gehringer M, Altmann K-H, Pluschke G (2017) The macrolide toxin mycolactone promotes bim-dependent apoptosis in Buruli ulcer through inhibition of mTOR. ACS Chem Biol 12(5):1297-1307. https://doi.org/10.1021/ acschembio. $7 b 00053$

15. Bolz M, Ruggli N, Ruf M-T, Ricklin ME, Zimmer G, Pluschke G (2014) Experimental infection of the pig with Mycobacterium ulcerans: a novel model for studying the pathogenesis of Buruli ulcer disease. PLoS Negl Trop Dis 8:e2968. https://doi.org/10.1371/journal. pntd.0002968

16. Ruf M-T, Bolz M, Vogel M, Bayi PF, Bratschi MW, Sopho GE, Yeboah-Manu D, Um Boock A, Junghanss T, Pluschke G (2016) Spatial distribution of Mycobacterium ulcerans in Buruli ulcer lesions: implications for laboratory diagnosis. PLoS Negl Trop Dis 10:e0004767. https:// doi.org/10.1371/journal.pntd.0004767

17. Addison NO, Pfau S, Koka E, Aboagye SY, Kpeli G, Pluschke G, Yeboah-Manu D, Junghanss $\mathrm{T}$ (2017) Assessing and managing wounds of Buruli ulcer patients at the primary and secondary health care levels in Ghana. PLoS Negl Trop Dis 11:e0005331. https://doi.org/10.1371/ journal.pntd.0005331

18. Amoussouhoui AS, Sopoh GE, Wadagni AC, Johnson RC, Aoulou P, Agbo IE, Houezo J-G, Boyer M, Nichter M (2018) Implementation of a decentralized community-based treatment program to improve the management of Buruli ulcer in the Ouinhi district of Benin, West Africa. PLoS Negl Trop Dis 12:e006291. https://doi.org/10.1371/journal.pntd.0006291

19. Aboagye SY, Asare P, Otchere ID, Koka E, Mensah GE, Yirenya-Tawiah D, Yeboah-Manu D (2017) Environmental and behavioral drivers of Buruli ulcer disease in selected communities along the Densu River basin of Ghana: a case-control study. Am J Trop Med Hyg 96:10761083. https://doi.org/10.4269/ajtmh.16-0749

20. Ampah KA, Nickel B, Asare P, Ross A, De-Graft D, Kerber S, Spallek R, Singh M, Pluschke G, Yeboah-Manu D, Röltgen K (2016) A sero-epidemiological approach to explore transmission of Mycobacterium ulcerans. PLoS Negl Trop Dis 10:e0004387. https://doi.org/10.1371/ journal.pntd.0004387 
21. Williamson HR, Mosi L, Donnell R, Aqqad M, Merritt RW, Small PLC (2014) Mycobacterium ulcerans fails to infect through skin abrasions in a guinea pig infection model: implications for transmission. PLoS Negl Trop Dis 8:e2770. https://doi.org/10.1371/journal.pntd.0002770

22. Sarfo FS, Lavender CJ, Fyfe JAM, Johnson PDR, Stinear TP, Phillips RO (2011) Mycobacterium ulcerans DNA not detected in faecal samples from Buruli ulcer patients: results of a pilot study. PLoS One 6:e19611. https://doi.org/10.1371/journal.pone.0019611

23. Bolz M, Bratschi MW, Kerber S, Minyem JC, Um Boock A, Vogel M, Bayi PF, Junghanss T, Brites D, Harris SR, Parkhill J, Pluschke G, Lamelas Cabello A (2015) Locally confined clonal complexes of Mycobacterium ulcerans in two Buruli ulcer endemic regions of Cameroon. PLoS Negl Trop Dis 9:e0003802. https://doi.org/10.1371/journal.pntd.0003802

24. Doig KD, Holt KE, Fyfe JAM, Lavender CJ, Eddyani M, Portaels F, Yeboah-Manu D, Pluschke G, Seemann T, Stinear TP (2012) On the origin of Mycobacterium ulcerans, the causative agent of Buruli ulcer. BMC Genomics 13:258. https://doi.org/10.1186/1471-2164-13-258

25. Eddyani M, Vandelannoote K, Meehan CJ, Bhuju S, Porter JL, Aguiar J, Seemann T, Jarek M, Singh M, Portaels F, Stinear TP, de Jong BC (2015) A genomic approach to resolving relapse versus reinfection among four cases of Buruli ulcer. PLoS Negl Trop Dis 9:e0004158. https:// doi.org/10.1371/journal.pntd.0004158

26. Vandelannoote K, Meehan CJ, Eddyani M, Affolabi D, Phanzu DM, Eyangoh S, Jordaens K, Portaels F, Mangas K, Seemann T, Marsollier L, Marion E, Chauty A, Landier J, Fontanet A, Leirs H, Stinear TP, de Jong BC (2017) Multiple introductions and recent spread of the emerging human pathogen Mycobacterium ulcerans across Africa. Genome Biol Evol 9:414-426. https://doi.org/10.1093/gbe/evx003

27. Awah PK, Boock AU, Mou F, Koin JT, Anye EM, Noumen D, Nichter M, Stop Buruli Consortium (2018) Developing a Buruli ulcer community of practice in Bankim, Cameroon: a model for Buruli ulcer outreach in Africa. PLoS Negl Trop Dis 12:e0006238. https://doi. org/10.1371/journal.pntd.0006238

28. Amoussouhoui AS, Johnson RC, Sopoh GE, Agbo IE, Aoulou P, Houezo J-G, Tingbe-Azalou A, Boyer M, Nichter M (2016) Steps toward creating a therapeutic community for inpatients suffering from chronic ulcers: lessons from Allada Buruli Ulcer Treatment Hospital in Benin. PLoS Negl Trop Dis 10:e0004602. https://doi.org/10.1371/journal.pntd.0004602

29. Ahorlu CK, Koka E, Yeboah-Manu D, Lamptey I, Ampadu E (2013) Enhancing Buruli ulcer control in Ghana through social interventions: a case study from the Obom sub-district. BMC Public Health 13:59. https://doi.org/10.1186/1471-2458-13-59

Open Access This chapter is licensed under the terms of the Creative Commons Attribution 4.0 International License (http://creativecommons.org/licenses/by/4.0/), which permits use, sharing, adaptation, distribution and reproduction in any medium or format, as long as you give appropriate credit to the original author(s) and the source, provide a link to the Creative Commons license and indicate if changes were made.

The images or other third party material in this chapter are included in the chapter's Creative Commons license, unless indicated otherwise in a credit line to the material. If material is not included in the chapter's Creative Commons license and your intended use is not permitted by statutory regulation or exceeds the permitted use, you will need to obtain permission directly from the copyright holder.

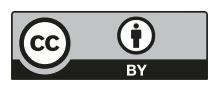

